\title{
When are projections also embeddings?
}

\author{
I. M. Moroz*, C. Letellier† \& R. Gilmore†† \\ * Mathematical Institute, 24-29 St Giles', Oxford OX1 3LB, UK \\ $\dagger$ Analyse TOpologique et de MOdélisation de SYstèmes Dynamiques \\ Université de Rouen - CORIA UMR 6614, BP 12, \\ F-76801 Saint-Etienne du Rouvray cedex, France \\ $\ddagger$ Physics Department, Drexel University, Philadelphia, Pennsylvania 19104, USA
}

(Dated: Physical Review E, to be submitted, December 12, 2006)

\begin{abstract}
We study an autonomous four-dimensional dynamical system used to model certain geophysical processes. This system generates a chaotic attractor that is strongly contracting, with four Lyapunov exponents $\lambda_{i}$ that satisfy $\lambda_{1}+\lambda_{2}+\lambda_{3}<0$, so the Lyapunov dimension is $D_{L}=2+\left|\lambda_{3}\right| / \lambda_{1}<3$ in the range of coupling parameter values studied. As a result, it should be possible to find three-dimensional spaces in which the attractors can be embedded so that topological analyses can be carried out to determine which stretching and squeezing mechanisms generate chaotic behavior. We study mappings into $R^{3}$ to determine which can be used as embeddings to reconstruct the dynamics. We find dramatically different behavior in the two simplest mappings: projections from $R^{4}$ to $R^{3}$. In one case the one-parameter family of attractors studied remains topologically unchanged for all coupling parameter values. In the other case, during an intermediate range of parameter values the projection undergoes self-intersections, while the embedded attractors at the two ends of this range are topologically mirror images of each other.
\end{abstract}

PACS numbers: 05.45.-a

\section{INTRODUCTION}

Ten years ago Hide, Skeldon and Acheson [1] introduced a model for a self-exciting Faraday disk dynamo as a simple analogue for the heat storage capacity in the oceans, thought to be a key factor in the dynamical processes underlying the El Niño Southern Oscillation. They noted that the equivalence of a capacitor to a motor as a circuit element opened up the relevance of the dynamo to the geomagnetic field.

Self-exciting Faraday disk dynamos are of interest since they contain some of the key ingredients of large-scale naturally-occurring MHD dynamos, while being of considerably lower dimension and therefore more amenable to systematic study. Many of the low-order dynamo models of this family have rich ranges of behaviour with irregular reversals a common feature, as well as steady, periodic and coexisting states (due to hysteresis effects). What has been lacking has been a means of distinguishing between these and other models as a prelude to comparing them with the large-scale counterparts.

Recent investigations of several extensions to this original model [2-6] have shown the classic Lorenz equations to result in a special limit when one of the key bifurcation parameters, $\beta$, which measures the inverse moment of inertia of the armature of the dynamo motor, vanishes. In this paper we focus on one of these models [3], which we term the extended Malkus-Robbins dynamo (or EMR), since it reduces to the Malkus-Robbins equations
[7] when $\beta=0$.

The dynamical system that is studied is presented in Sect. II. It is a Lorenz system coupled linearly to an external variable whose behavior is coupled back into the Lorenz system. It is a four-dimensional dynamical system with an internal order-two symmetry. The principal question we wish to address is: what stretching and squeezing processes are responsible for the dynamics, and how do they change as the coupling strength changes? Questions of this type can be addressed for three-dimensional chaotic attractors, for which topological analyses can be carried out.

An analysis of the spectrum of Lyapunov exponents reveals that for sufficiently strong damping on the external variable $\Lambda$ the system is strongly contracting, with Lyapunov dimension $D_{L}<3$, so that in principal there is a three-dimensional manifold in which the attractor can be embedded. As a result the attractor is three-dimensional and a topological analysis can be carried out if a suitable embedding can be found. Such an analysis is based on the unstable periodic orbits associated with the attractor. The method of treating these orbits is described in Sec. III. In Sec. IV we briefly review how linking numbers of unstable periodic orbits are computed in a three-dimensional embedding. In Sec. V we review the two simplest mappings, which are projections into threedimensional subspaces, and in Sec. VI we perform topological analyses in both projections. These analyses show one projection is an embedding for all values of the cou- 
pling parameter, the other only for extreme (small, large) values of the coupling parameter. For intermediate values the projected attractor exhibits self intersections, and in fact turns itself into its mirror image during the transition from small to large values of this parameter. In Sec. VII we introduce a number of other $1 \rightarrow 1$ mappings into three-dimensional phase spaces, and in Sec. VIII we introduce two $2 \rightarrow 1$ mappings into three-dimensional phase spaces. Topological analyses were carried out on images of all unstable periodic orbits up to period six in each of these phase spaces. Results are summarized in Table III. Some mappings are embeddings and others are not because of the nature of the coupling between the variables. This coupling also has a direct impact on the observability of a nonlinear system. The correlation between embeddings and observability is presented in Sec. IX. In Sec. $\mathrm{X}$ we describe typical Poincaré surfaces of section in both $1 \rightarrow 1$ mappings and $2 \rightarrow 1$ mappings, and in Sec. XI we present the associated return maps. We summarize our results in Sec. XII. Topological analysis of all embeddings of the attractors reveals a Lorenz stretching and tearing mechanism at work to create the chaotic attractor.

\section{DYNAMICAL SYSTEM EQUATIONS}

The extended Malkus-Robbins dynamo equations are

$$
\left\{\begin{array}{l}
\dot{X}=\sigma(Y-X)-\hat{\beta} U \\
\dot{Y}=\frac{R X}{\nu}-Y-X Z \\
\dot{Z}=-\nu Z+X Y \\
\dot{U}=X-\Lambda U
\end{array}\right.
$$

with $\hat{\beta}=7.1111 \beta$. For further details of the meanings of the variables and the positive parameters, see [3]. This four-dimensional system is equivariant, that is, satisfies the symmetry

$$
\Gamma \cdot \boldsymbol{f}(\boldsymbol{X})=\boldsymbol{f}(\Gamma \cdot \boldsymbol{X}) .
$$

The equivariance matrix $\Gamma$ is

$$
\Gamma=\left[\begin{array}{cccc}
-1 & 0 & 0 & 0 \\
0 & -1 & 0 & 0 \\
0 & 0 & +1 & 0 \\
0 & 0 & 0 & -1
\end{array}\right]
$$

This defines a symmetry in $R^{4}$ which is a rotation about the $Z$-axis: $\mathcal{R}_{Z}(\pi)$. We will investigate in more detail two three-dimensional subsets of variables, each inheriting a different symmetry from Eq. (3). These are the sets $(X, Y, Z)$ and $(X, Y, U)$ with symmetries

$$
\begin{array}{lccc}
(X, Y, Z) \rightarrow(-X,-Y,+Z) & R_{Z}(\pi) & \text { Rotation } \\
(X, Y, U) \rightarrow(-X,-Y,-U) & P & \text { Inversion }
\end{array}
$$

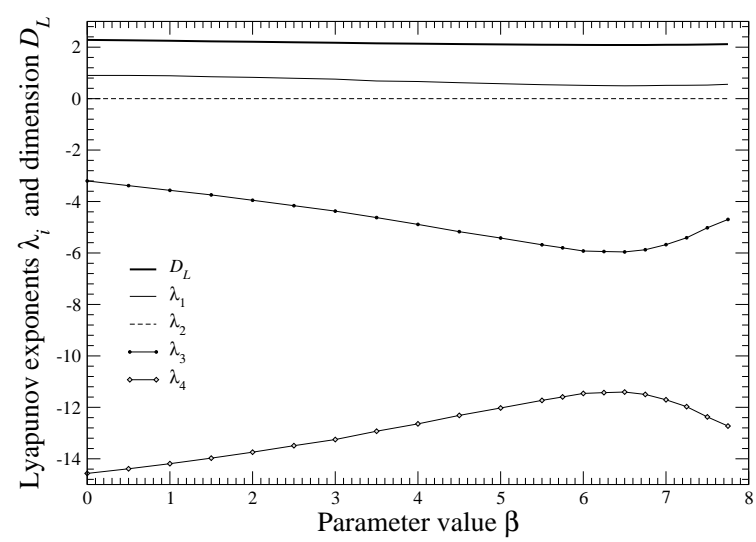

FIG. 1: Plot of the four Lyapunov exponents $\lambda_{j}$ and the Lyapunov dimension $D_{L}$ for the chaotic attractor generated by Eq. (1) as a function of $\beta$. For these values the attractor is effectively three-dimensional. Parameter values: $(\sigma, \nu, R, \Lambda)=(10,8 / 3,74.667,3.2)$.

For $\beta=0$ this set of equations describes the Lorenz dynamical system driving a linear dynamical system $U$ with damping $\Lambda$. There is no feedback since the $U$ equation decouples, and the $(X, Y, Z)$ subsystem is precisely the Lorenz system. For $\beta \neq 0$ the driven subsystem provides feedback into the Lorenz system. We expect that for small values of the coupling constant the threedimensional subsystem $(X, Y, Z)$ will behave very much like the Lorenz system, but for larger values of the coupling, departures from Lorenz-like behavior will become evident. As a result, the dynamical system was studied as a function of the coupling strength $\beta$.

As a first step in the analysis of this system we chose the Lorenz parameters as $(\sigma, \nu, R)=(10,8 / 3,74.667)$ so as to produce chaotic attractor for $\beta=0$. We also fixed the damping parameter for $U$ at $\Lambda=3.2$. A bifurcation diagram for the system was computed as a function of $\beta$ in the range $0 \leq \beta \leq 7.9$, at which point a boundary crisis destroys the attractor [5]. The bifurcation diagram showed that the chaotic attractor persists without windows for this range of $\beta$ values.

As a second step we computed the Lyapunov exponents and the Lyapunov dimension as a function of $\beta$. The results are shown in Fig. 1. In this range one Lyapunov exponent is positive, one remains approximately zero, and two are negative. The Lyapunov exponents limit at $\beta=0$ to those of the Lorenz attractor $(0.9027,0.0,-14.5691)$ including $\lambda_{3}=-\Lambda=-3.2$.

For all values of $\beta$ in this range the system is strongly contracting. This means that the sum of the three largest Lyapunov exponents is negative. As a consequence the Lyapunov dimension $D_{L}$ is less than three. The KaplanYorke estimate for $D_{L}=2+\lambda_{1} /\left|\lambda_{3}\right|$ is also shown in Fig. 1. This statistic ranges between $2.08<D_{L}<2.29$. Since the Lyapunov dimension is less than three, this system is effectively three-dimensional. This means that it should 
be possible to find a three-dimensional manifold in $R^{4}$ that contains the attractor. We point out in passing that as $\Lambda$ decreases below the value of the positive Lyapunov exponent $\lambda_{1}=0.9027$ the Lyapunov dimension of the attractor increases above 3.0, at least for small values of $\beta$.

\section{PERIODIC ORBITS}

Since the attractor is effectively three-dimensional for all values of $\beta$ studied, a topological analysis can be carried out to determine the mechanism generating chaotic behavior provided a three-dimensional embedding can be found. We anticipate that the mechanism observed will be the same as the mechanism generating the Lorenz attractor, but the representation of this mechanism may depend on the embedding chosen [17].

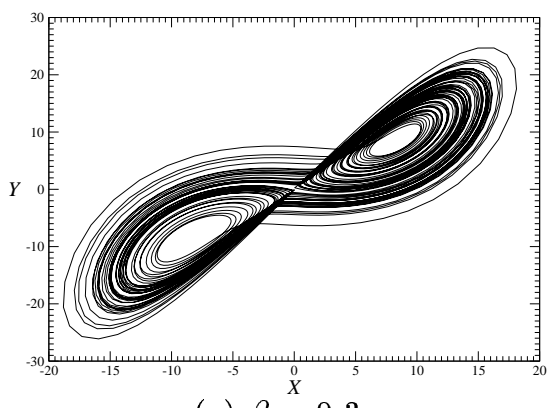

(a) $\beta \stackrel{x}{=} 0.3$

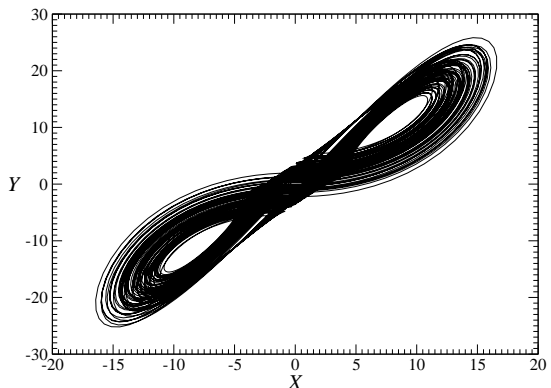

(b) $\beta=3.8$

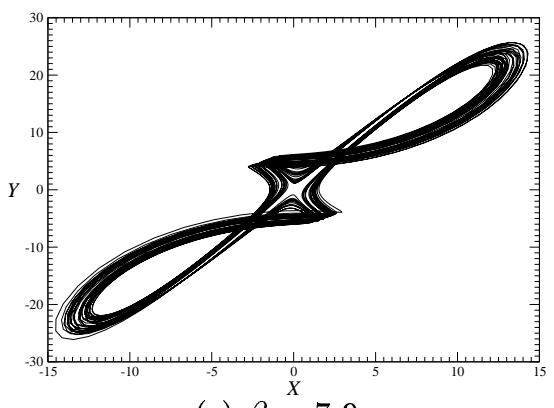

(c) $\beta=7.9$

FIG. 2: $X-Y$ plane projection of the chaotic attractors generated by the EMR equations Eq. (1) for different $\beta$ values. Parameter values: $(\sigma, \nu, R, \Lambda)=$ $(10,8 / 3,74.667,3.2)$.
A topological analysis is based on the unstable periodic orbits that exist in abundance in chaotic attractors. To perform this analysis it is sufficient to find only a relatively small number of these orbits. The method of close returns was used in $R^{4}$ to locate surrogates for unstable periodic orbits. These orbits, in fact the entire unstable trajectory, could be labeled by two symbols, $L$ and $R$, indicating passage in the neighborhood of the left or right hand focus. The two foci are clearly evident in Fig. 2, which shows projections of the strange attractors from $R^{4}$ into the $X-Y$ plane, for three values of $\beta$. Most of the orbits up to period six are present in the chaotic attractor generated for all values of the parameter $\beta$. The two period-one orbits $L$ and $R$ are not present. We found the period-two orbit $L R$, two period-three orbits, three period-four orbits, and six period-five orbits. This indicates that the topological entropy of this attractor is very high, slightly less than $\log 2$.

In the topological analyses to be described, these orbits in $R^{4}$ were mapped into the spaces used for embedding attempts.

\section{LINKING NUMBERS}

Topological analyses proceed by determining how the unstable periodic orbits in a chaotic attractor are organized with respect to each other. This organization is determined by the linking numbers of pairs of periodic orbits $[8,9]$. Linking numbers can be determined by computing a Gauss integral. However, they are more simply computed by projecting the two orbits from the three-dimensional space in which they exist onto a two dimensional surface. The orbits do not intersect in three dimensions: this would violate the uniqueness condition. However, they appear to intersect in the projection. Each intersection is given a sign \pm 1 . The sign is determined as follows. Arrows indicating the flow direction are assigned to the two crossing segments. The arrow closer to the observer is rotated into the other arrow through the smallest possible angle. If the rotation is right-handed the crossing is assigned the crossing number +1 ; if lefthanded the crossing is -1 . The linking number is half the sum of the signed crossings of one orbit with the other. In Fig. 3 two orbits are shown. These are extracted from a chaotic attractor generated by Eq. (1) and projected into a three-dimensional space. In this figure the five positive crossings are indicated by + and the single negative crossing is indicated by a $\bullet$. The linking number of this pair of orbits is therefore +2 .

In four dimensions periodic orbits are not rigidly organized. Knots "fall apart" in four and higher dimensions. For this reason the topological analyses that are carried out here are restricted to three-dimensional spaces. 


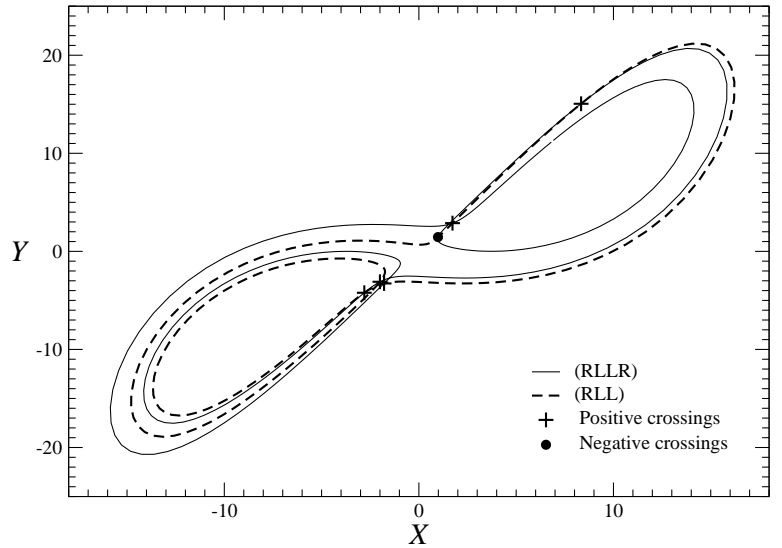

FIG. 3: Two linked orbits are projected onto a twodimensional surface. Positive crossings are indicated by + and the negative crossing by a $\bullet$. The linking number of the orbits is half the sum of signed crossings: $L N(R L L R, R L L)=\frac{1}{2}(+5-1)=+2$. Parameter values: $(\sigma, \nu, R, \Lambda)=(10,8 / 3,74.667,3.2)$ and $\beta=$ ?.

\section{MAPPINGS INTO THREE-DIMENSIONAL SPACES}

Before a topological analysis can be carried out, an embedding of the attractor into a three-dimensional space must be constructed. Many mappings are possible, but two are essentially trivial. These are simple projections from $R^{4}$ to $R^{3}$ obtained by "forgetting" one of the four coordinates. Two projections suggest themselves:

$$
\begin{array}{cccc}
R^{4} & \rightarrow & R^{3} & \text { Symmetry } \\
\hline(X, Y, Z, U) & \rightarrow(X, Y, Z) & R_{Z}(\pi) \\
(X, Y, Z, U) & \rightarrow(X, Y, U) & P
\end{array}
$$

Projections into the $X-Y$ subspace from either of these three-dimensional projections are shown in Fig. 2 for three values of $\beta$. These figures show that as $\beta$ increases the attractor undergoes increasing curvature in the neighborhood of the origin.

Other possible mappings include differential mappings based on a single variable, for example $X(t) \rightarrow$ $(X(t), \dot{X}(t), \ddot{X}(t))$. Similar mappings based on the other variables are also possible. We finally also considered $2 \rightarrow 1$ mappings. These are treated in Sec. VIII.

\section{TOPOLOGICAL ANALYSES ON PROJECTIONS}

A topological analysis was carried out on the projection of the chaotic attractor into each of the two threedimensional subspaces suggested in Eq. (5).

The first topological analysis was carried out on the projection $(X, Y, Z, U) \rightarrow(X, Y, Z)$. Periodic orbits that were identified in $R^{4}$ were projected into this subspace and their linking numbers were computed. This was done by counting crossings in their projection to the $X-Y$ plane. The orbits were expected to be organized as they are in the Lorenz dynamical system, at least for small values of $\beta$. The linking numbers of the projected orbits were compared with those in the Lorenz dynamical system, and described by the Lorenz branched manifold with rotation symmetry. This branched manifold is shown in Fig. 4(a). The linking numbers of periodic orbits on this branched manifold are summarized in Table I [10].

TABLE I: Linking numbers of orbits in the Lorenz branched manifold with rotation symmetry, up to period four. In the range $0 \leq \beta<0.6$, linking numbers of periodic orbits in the projected attractor exhibit these values. In the range $5.4<\beta<7.9$ linking numbers are the negatives of these values.

\begin{tabular}{c|cccccc} 
& LR & LLR & LRR & LLLR & LRRR & LLRR \\
\hline$L R$ & - & 1 & 1 & 1 & 1 & 2 \\
$L L R$ & 1 & - & 1 & 2 & 1 & 2 \\
$L R R$ & 1 & 1 & - & 1 & 2 & 2 \\
LLLR & 1 & 2 & 1 & - & 1 & 2 \\
LRRR & 1 & 1 & 2 & 1 & - & 2 \\
LLRR & 2 & 2 & 2 & 2 & 2 & -
\end{tabular}

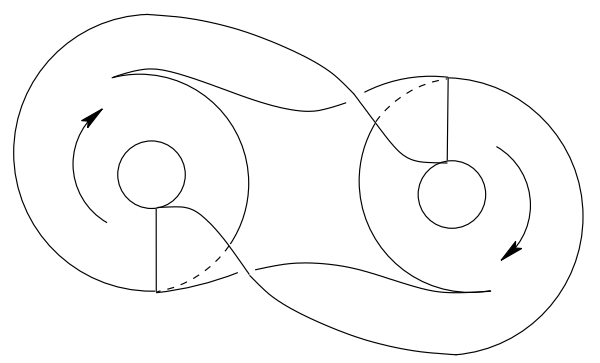

(a) Rotation symmetry

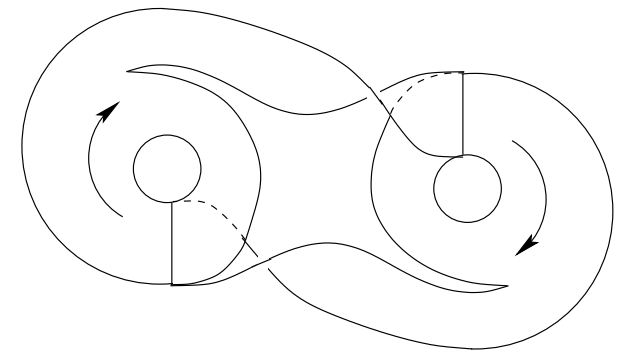

(b) Inversion symmetry

FIG. 4: Branched manifolds for the Lorenz attractor with (a) rotation and (b) inversion symmetry. Branched manifolds describe the topological organization of all the unstable periodic orbits in a chaotic attractor. The two branched manifolds shown are "group continuations" of one another [10].

For $0 \leq \beta<0.6$ the linking numbers of the projected 
TABLE II: Linking numbers of orbits in the Lorenz branched manifold with inversion symmetry.

\begin{tabular}{c|cccccc} 
& LR & LLR & LRR & LLLR & LRRR & LLRR \\
\hline$L R$ & - & 0 & 0 & 0 & 0 & 0 \\
$L L R$ & 0 & - & 0 & +1 & 0 & +1 \\
$L R R$ & 0 & 0 & - & 0 & -1 & -1 \\
$L L L R$ & 0 & +1 & 0 & - & 0 & +1 \\
$L R R R$ & 0 & 0 & -1 & 0 & - & -1 \\
$L L R R$ & 0 & +1 & -1 & +1 & -1 & -
\end{tabular}

orbits were compatible with the integers in this table. In the range $0.6<\beta<5.4$ we found that the table of linking numbers obtained from the periodic orbits was not compatible with any brnached manifold. Furthermore, each linking number systematically decreased by integer steps through zero, finally assuming the negative of its value in the small $\beta$ region. In the range $5.4<\beta<7.9$ the table of linking numbers was the negative of that shown in Table I. As a consequence, the branched manifolds describing the projections of the chaotic attractor in $R^{4}$ into the $(X, Y, Z)$ subspace are mirror images of each other in the two disconnected ranges of $\beta$, where the projection is an embedding. Fig. 4(a) and its mirror image both exhibit rotation symmetry.

Images of the chaotic attractor, projected into the three-dimensional space with coordinates $(X, Y, Z)$, are shown in Fig. 5. The orientation has been chosen to enable visualization of the region of self-intersection. The projected attractor changes handedness during the transition through the region of self-intersections (in the projection) from small to large values of $\beta$.

The projection $(X, Y, Z, U) \rightarrow(X, Y, U)$ into the subspace where the attractor has inversion symmetry showed entirely different behavior. The branched manifold for the Lorenz attractor with inversion symmetry is shown in Fig. 4(b). In this projection the linking numbers should have values given in Table II. The periodic orbits, projected from $R^{4}$ into this subspace, had linking numbers compatible with this table for all values of $\beta$. We were thus able to conclude, through topological analysis methods, that the projection $(X, Y, Z, U) \rightarrow(X, Y, U)$ is an embedding for all values of $\beta$ but that the projection $(X, Y, Z, U) \rightarrow(X, Y, Z)$ is an embedding only for some values of $\beta$. When there was an embedding, the mechanism exhibited (tearing and squeezing) is the mechanism that operators to generate chaotic behavior for the Lorenz attractor at comparable parameter values.

\section{ADDITIONAL MAPPINGS}

We studied a number of other mappings to determine if they were embeddings. These included differential mappings based on each of the four coordinates. Under the

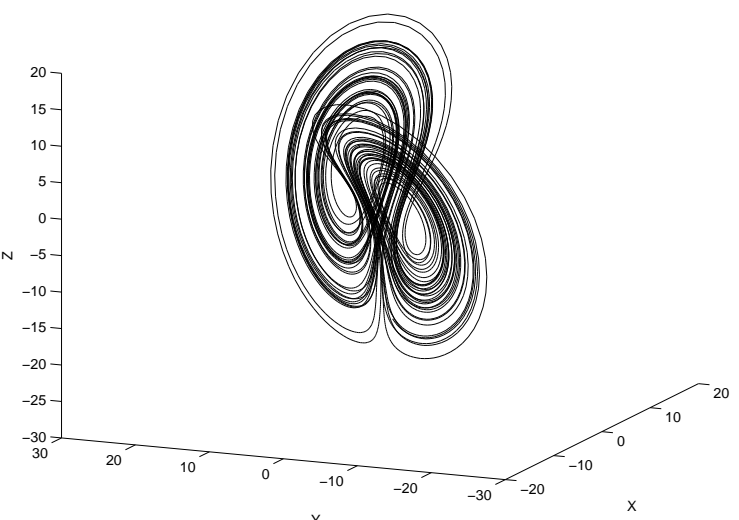

(a) $\beta=0.3$

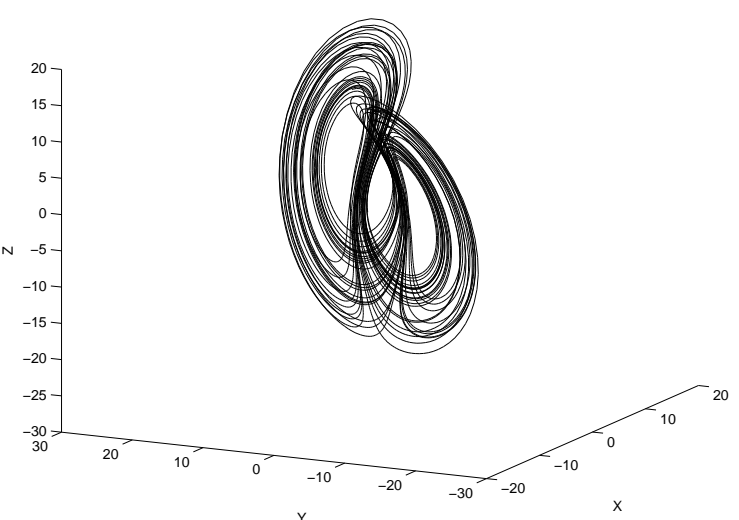

(b) $\beta=3.8$

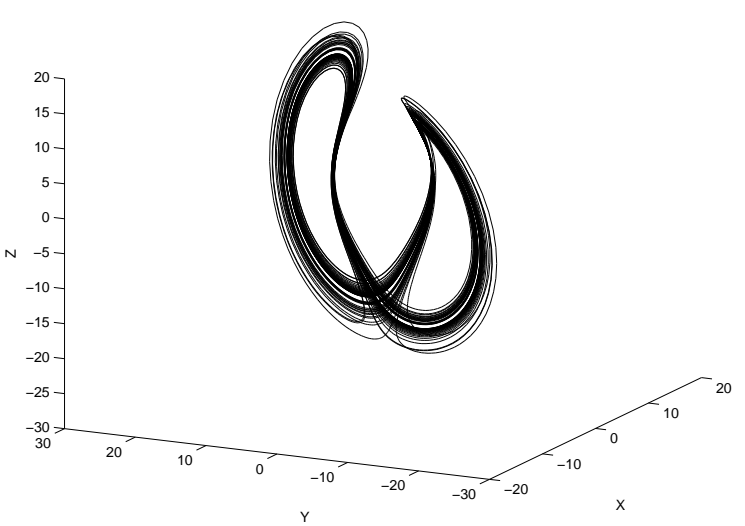

(c) $\beta=7.9$

FIG. 5: Chaotic attractor projected from $R^{4}(X, Y, Z, U)$ into $R^{3}(X, Y, Z)$ for three values of the parameter $\beta$. Selfintersections are evident for $\beta=3.8$. The two projected attractors for $\beta=0.3$ and $\beta=7.9$ are described by branched manifolds that are mirror images of each other. 
mappings based on the antisymmetric coordinates $X, Y$, and $U$, the strange attractor exhibited an inversion symmetry, whether or not the mapping was an embedding. The linking numbers of the mapped orbits were compared with those shown in Table II. Restricted differential embeddings of the form $(V, \dot{V}, Z)$, where the variable $V$ was chosen to be each of the "antisymmetric" variables $X, Y$, and $U$, were also studied. These exhibited rotation $\left(R_{Z}\right)$ symmetry. Mappings of the strange attractor into the two three-dimensional spaces $(X, \dot{X}, \ddot{X})$ and $(X, \dot{X}, Z)$ can be projected to the same two-dimensional subspace $(X, \dot{X})$. This projection is shown in Fig. 6(a). Projections into the $(Y, \dot{Y})$ and $(U, \dot{U})$ subspaces are also shown in this figure.

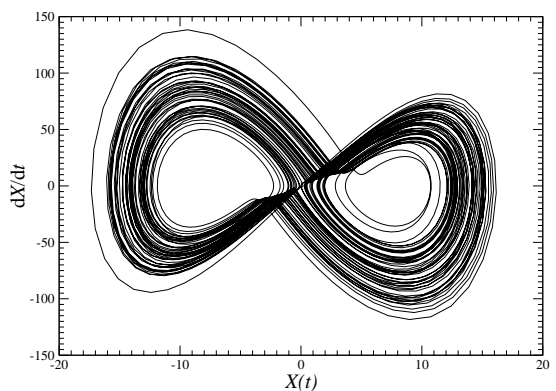

$X-\dot{X}$ plane projection

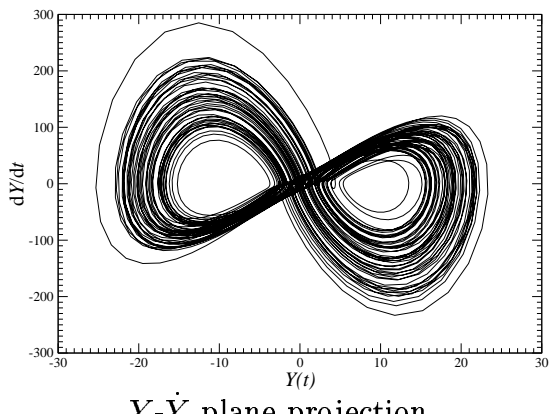

$Y-\dot{Y}$ plane projection

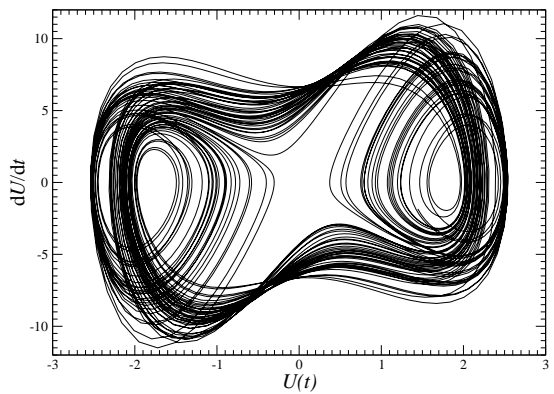

$U-\dot{U}$ plane projection

FIG. 6: Differential embeddings based on the odd variables $X, Y$, and $U$. Parameter values: $(\sigma, \nu, R, \Lambda)=$ $(10,8 / 3,74.667,3.2)$.

The periodic orbits in $R^{4}$ were mapped into threedimensional phase spaces under each of the six mappings of the type $(V, \dot{V}, \ddot{V})$ and $(V, \dot{V}, Z)$, with $V$ an odd variable $X, Y$, or $U$. Tables of linking numbers were com- puted for these mapped orbits for various values of the parameter $\beta$. The mappings depending on $X$ and $U$ provided embeddings for all values of $\beta$. The two mappings depending on $Y$ did not provide embeddings for $\beta$ in the range $0.6<\beta<5.4$. These results are summarized in Table III.

TABLE III: Variables used in searches for embeddings of the chaotic attractor generated by Eq. (1). If the mapping failed to be an embedding, it did so in the range $0.6 \leq \beta \leq 5.4$.

\begin{tabular}{ccc}
\hline \hline Variables & Type & Embedding? \\
\hline$(X, Y, Z)$ & $1 \rightarrow 1$ & $\mathrm{~N}$ \\
$(X, Y, U)$ & $1 \rightarrow 1$ & $\mathrm{Y}$ \\
$(X, \dot{X}, \ddot{X})$ & $1 \rightarrow 1$ & $\mathrm{Y}$ \\
$(Y, \dot{Y}, \ddot{Y})$ & $1 \rightarrow 1$ & $\mathrm{~N}$ \\
$(Z, \dot{Z}, \ddot{Z})$ & $2 \rightarrow 1$ & $\mathrm{Y}$ \\
$(U, \dot{U}, \ddot{U})$ & $1 \rightarrow 1$ & $\mathrm{Y}$ \\
$(X, \dot{X}, Z)$ & $1 \rightarrow 1$ & $\mathrm{Y}$ \\
$(Y, \dot{Y}, Z)$ & $1 \rightarrow 1$ & $\mathrm{~N}$ \\
$(U, \dot{U}, Z)$ & $1 \rightarrow 1$ & $\mathrm{Y}$ \\
$\left(X^{2}-Y^{2}, 2 X Y, Z\right)$ & $2 \rightarrow 1$ & $\mathrm{~N}$ \\
\hline \hline
\end{tabular}

\section{IMAGE ATTRACTORS}

A differential mapping based on the even variable $Z$ cannot have the same topology as the embeddings based on the odd variables, as seen in Fig. 7. Rather, the attractor reconstructed from this variable (when this mapping is an embedding) is a $2 \rightarrow 1$ image of the chaotic attractor generated by Eqs. (1).

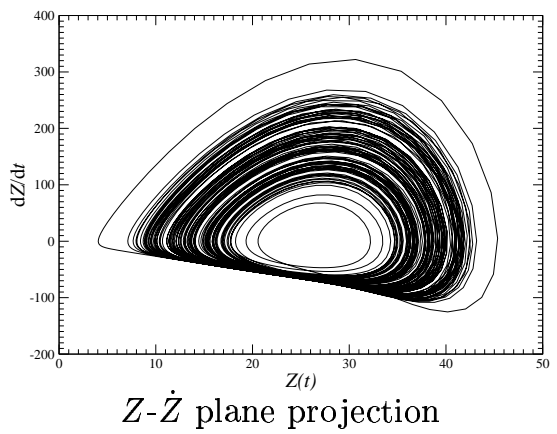

FIG. 7: Differential embedding based on the even variable $Z$. Parameter values: $(\sigma, \nu, R, \Lambda)=$ $(10,8 / 3,74.667,3.2)$ and $\beta=3.8$.

Other $2 \rightarrow 1$ images can be constructed. One that has often been used is $(X, Y, Z, U) \rightarrow\left(X^{2}-Y^{2}, 2 X Y, Z\right)$ $[10-12]$. This attractor is shown in Fig. 8 as it evolves 
under change of the control parameter $\beta$. Other $2 \rightarrow 1$ images in $R^{3}$ can be obtained by replacing $X$ or $Y$ by $U$. In addition, $2 \rightarrow 1$ images can also be obtained from $(X, Y, U)$ by modding out the inversion symmetry.

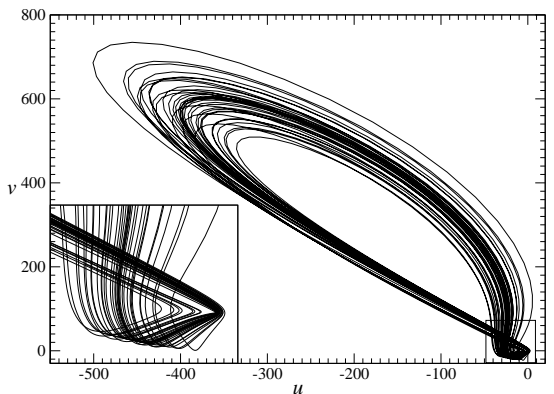

(a) $\beta=7.9$

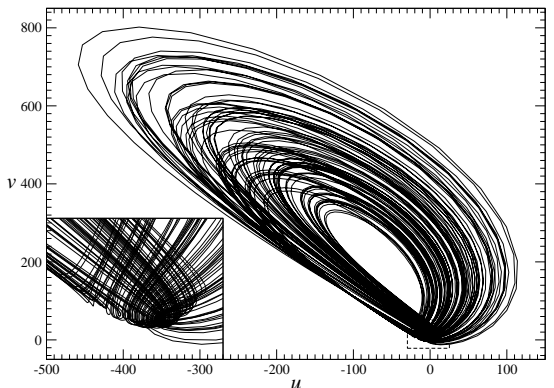

(b) $\beta=3.8$

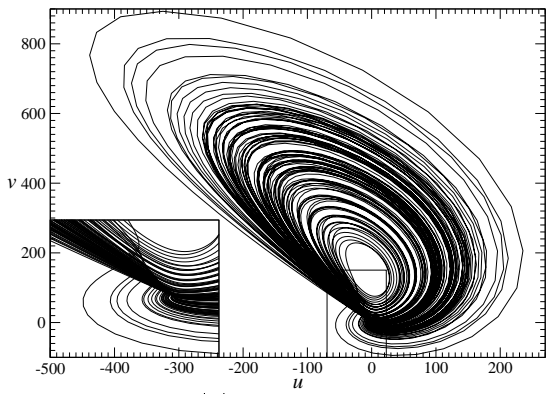

(c) $\beta=0.3$

FIG. 8: Plane projections of the image attractors $(X, Y, Z, U) \rightarrow(u, v, Z)$ with $u=X^{2}-Y^{2}, v=2 X Y$, for three values of $\beta$. A great deal of convulsion takes place in the neighborhood of the origin. Parameter values: $(\sigma, \nu, R, \Lambda)=(10,8 / 3,74.667,3.2)$.

Periodic orbits in $R^{4}$ were mapped into periodic orbits in the $2 \rightarrow 1$ image attractors. The image orbits are also described by two symbols, 0 and 1 , obtained from the symbolic dynamics based on $L$ and $R$ of their covering orbits in $R^{4}$ by the following algorithm:

$$
\begin{array}{ccccc}
\text { In } R^{4} & L \rightarrow L & R \rightarrow R & L \rightarrow R & R \rightarrow L \\
\text { In image } & 0 & 0 & 1 & 1
\end{array}
$$

For example, the periodic orbit $L L L R$ in $R^{4}$ maps to a periodic orbit with symbol name 0011 in the image. Linking numbers for periodic orbits up to period four
TABLE IV: Linking numbers of periodic orbits in Smale horseshoe dynamics [8].

\begin{tabular}{c|ccccccc} 
& 1 & 01 & 001 & 011 & 0011 & 0001 & 0111 \\
\hline 1 & - & 1 & 1 & 1 & 1 & 1 & 2 \\
01 & 1 & - & 2 & 2 & 2 & 2 & 3 \\
001 & 1 & 2 & - & 3 & 3 & 3 & 4 \\
011 & 1 & 2 & 3 & - & 3 & 3 & 4 \\
0011 & 1 & 2 & 3 & 3 & - & 4 & 4 \\
0001 & 1 & 2 & 3 & 3 & 4 & - & 4 \\
0111 & 2 & 3 & 4 & 4 & 4 & 4 & -
\end{tabular}

were computed in the phase spaces generated by the embeddings $(Z, \dot{Z}, \ddot{Z})$ and $\left(X^{2}-Y^{2}, 2 X Y, Z\right)$ and compared with the linking numbers contained in Table IV over the range of values of the parameter $\beta$. We found that the first mapping was an embedding for all values of $\beta$ while the second failed to be an embedding in the parameter range $0.6<\beta<5.4$.

\section{OBSERVABILITY AND EMBEDDINGS}

The results collected in Table III show that one to one mappings depending on the variables $X$ or $U$ generally provide embeddings, while those depending on $Y$ and $Z$ sometimes do not. These observations are consistent with recent results in observability theory $[13,14]$.

A system is fully observable from a variable when it is possible to recover all the dynamical variables of the system. It has been shown that the observability of a system, that is, the quality with which the dynamics can be reconstructed from a measured variable, depends on the choice of the observable [13]. In particular, it was shown that the observability depends on the coupling between the dynamical variables. Basically, the lack of observability results from the nonlinearities which introduce a singular set between the original phase space and the reconstructed space, that is, a set of points of the original phase space which cannot be observed in the reconstructed space. Thus, it is possible to located where the nonlinearities are acting directly from a graph displaying the couplings between the variables as follows [14]. The interactions between the dynamical variables can be defined using the elements of the Jacobian matrix of the vector field $f_{i}\left(x_{j}\right)$, where $\dot{x}_{i}=f_{i}\left(x_{j}\right)$. Variable $x_{j}$ acts on variable $x_{i}$ when the term $J_{i j}$ of the Jacobian matrix is non-zero. This action is positive or negative depending on the sign of element $J_{i j}$. These interactions can be displayed as a graph. Each variable $x_{i}$ is represented by a node $N_{i}$. When the variable $j$ is present in the functions $f_{i}$, an arrow is drawn from node $N_{j}$ to node $N_{i}$. When the variable only appears in a linear term, the arrow is drawn with a solid line. If a variable appears in a nonlinear term, the arrow is drawn with a dashed line. 
The graph of interactions between the dynamical variables of the EMR dynamo equations is shown in Fig. 9. Using such a graphical representation, the best observable for a given set of equations can be identified without any analytical computation.

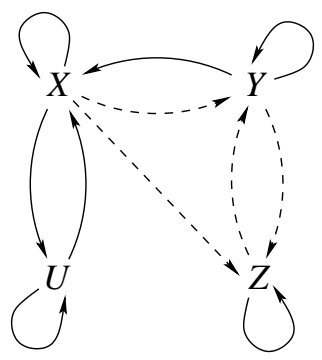

FIG. 9: Graph showing the couplings between the dynamical variables of the EMR dynamo equations.

When a variable is measured, it is known. Taking one of its successive time derivatives corresponds to moving along the arrows that reach this variable in the opposite direction, contrary to the arrow. In the case of the EMR dynamo equations, the Lyapunov dimension suggests that a three-dimensional embedding can be sufficient. This means that, depending on the observability associated with the "measured" variable, the dynamics could be embedded within a three-dimensional reconstructed space. For variables with a low observability, a higher dimensional phase space could be required. Using the graphical approach introduced in [14], it is possible to identify the best observable(s) as follows. Let us assume the $U$ variable is measured. Taking its first derivative allows us to reach the $X$ and $U$ variables but not the $Y$ and the $Z$ variables since there is no arrow from $N_{Y}$ to $N_{U}$ and from $N_{Z}$ to $N_{U}$ (Fig. 10). It is necessary to take the second derivative of $U$ to reach node $N_{Y}$ according to the arrow from $N_{Y}$ to $N_{X}$. Since all the arrows involved are associated with linear couplings, there is no need to compute the third derivative of $U$ to ensure having an embedding of the original four-dimensional dynamics. Indeed, the lack of nonlinearities in the second derivative $\ddot{U}$ prevents the existence of a singular set between the original four-dimensional phase space and the three-dimensional differential embedding induced by the $U$ variable. Since the nonlinearities are directly responsible for the lack of observability, the sooner the nonlinearities occur in the derivative, the less observable the dynamics is. For this reason, the dynamics underlying the EMR dynamo equations is less observable from the $Y$ or $Z$ variables than from the $X$ variable. In other words, a higher dimensional space can be required in the worst cases. As suggested by Table III, the lack of observability from the $Y$ variable is sufficiently important to forbid a three-dimensional embedding, even when the $Z$ variable is measured too.

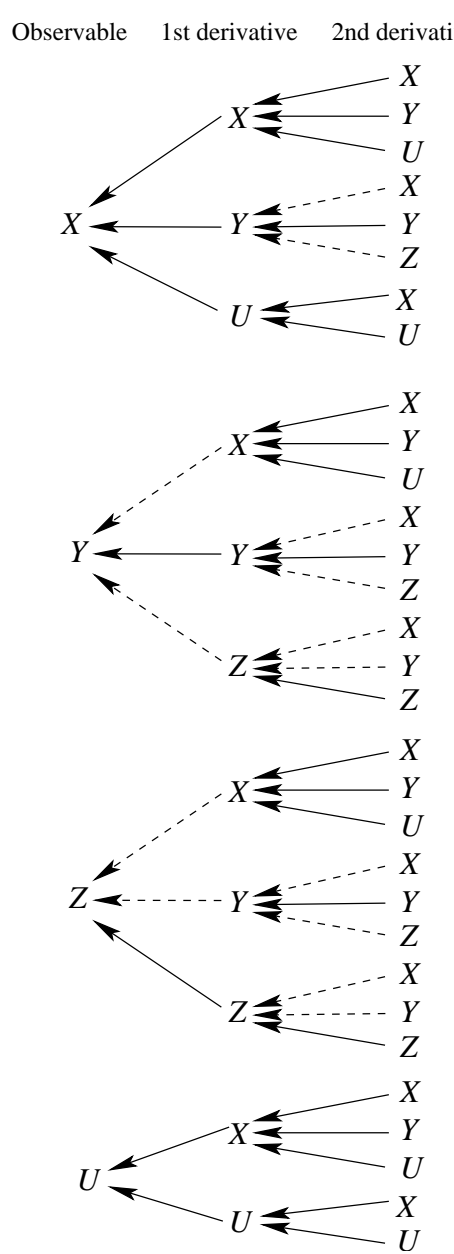

FIG. 10: Unfolded schematic view of the variables reached when the first and the second derivatives are computed. A nonlinearity in the first derivative (a dashed arrow between the observable and its first derivative) induces a more serious lack of observability than when a nonlinearity occurs in the second derivative (a dashed arrow between the first and the second derivatives of the observable).

\section{POINCARÉ SECTIONS}

Strictly speaking, Poincaré sections for the dynamical system Eq.(1) are three-dimensional. Since the chaotic attractor that is generated by these equations is threedimensional, it was useful to construct Poincaré sections in the three-dimensional phase spaces introduced by the mappings that are summarized in Table III. For the mappings whose projections are shown in Fig. 2, the global Poincaré surface of section consists of the union of two half planes $[15,16]$. In the case of the projection $(X, Y, Z, U) \rightarrow(X, Y, Z)$, one component of the global Poincaré surface of section can be chosen as the half-plane $(X=Y, Z)$ anchored on the line $X=Y \simeq 9.5$, which passes approximately through the focus. The other com- 
ponent is the mirror image half-plane. Similar arguments apply to the projection $(X, Y, Z, U) \rightarrow(X, Y, U)$ and the embeddings such as $(X, \dot{X}, \ddot{X})$, etc. The intersection of the attractor, projected into $(X, Y, Z)$, with the Poincaré surface of section is shown in Fig. 11(a). The form of this intersection remained basically unchanged for all values of the parameter $\beta$ in all the $1 \rightarrow 1$ mappings that were studied.

In the case of the $2 \rightarrow 1$ mappings, the image attractor exhibited a "hole in the middle." This means that the global Poincaré surface of section is connected. It consists of a single half-plane that is anchored on an axis passing through the focus. The intersection of the image attractor with such a half plane has the form shown in Fig. 12(a). This is clearly a $2 \rightarrow 1$ image of the intersection shown in Fig. 11(a). Again, the $V$ shape of this intersection was the same in both mappings and remained basically unchanged for all values of the parameter $\beta$. The most noticeable effect was that as $\beta$ increases from small to large values the separation between the two cusps in Fig. 12(b) increases linearly.

\section{FIRST RETURN MAPS}

Simple forward mapping of the intersections of the chaotic attractor with the Poincaré section produces results shown in Figs. 11(b) and 12(b). These are not strictly first return maps since the functions shown are not single valued.

In order to produce a more useful result we parameterized the $V$ shaped intersections with "geometric" coordinates. That is, we introduced a parameter, $s$, to measure position along the intersection of the attractor with the left half-plane in Fig. 11(c). This parameter decreased with $X$ linearly along the lower branch from $s=0$ at $X \simeq-11$ to $s=0.5$ at $X \simeq-17$, then increased along the upper branch form $s=0.5$ at the cusp to $s=1.0$ at $X \simeq-10.5$. A geometric parameter $t$ was introduced in a similar (symmetric) way to parameterize the intersection with the right hand branch. The first return map on the geometric parameters $s$ and $t$ is shown in Fig. 11(c). This is very similar to the first return map previously obtained for the Lorenz attractor.

A similar parameterization for the intersections of the $2 \rightarrow 1$ image attractors (c.f., Fig. 12(b)) was also carried out. The return map on that parameter is shown in Fig. 12(c) for the $\left(X^{2}-Y^{2}, 2 X Y, Z\right)$ mapping. The other $2 \rightarrow 1$ mapping $(Z, \dot{Z}, \ddot{Z})$ was similar. As $\beta$ was increased, the return map changed in two ways. The singularity in the slope at $s=0.5$ became less pronounced, and the regions of near vertical slope (extreme instability) moved outward, to larger values of $|s-0.5|$. At the same time the spectrum of unstable periodic orbits remained almost unchanged. There was almost a complete symbolic dynamics on two symbols. This indicates that the topological entropy of these attractors, as well as the

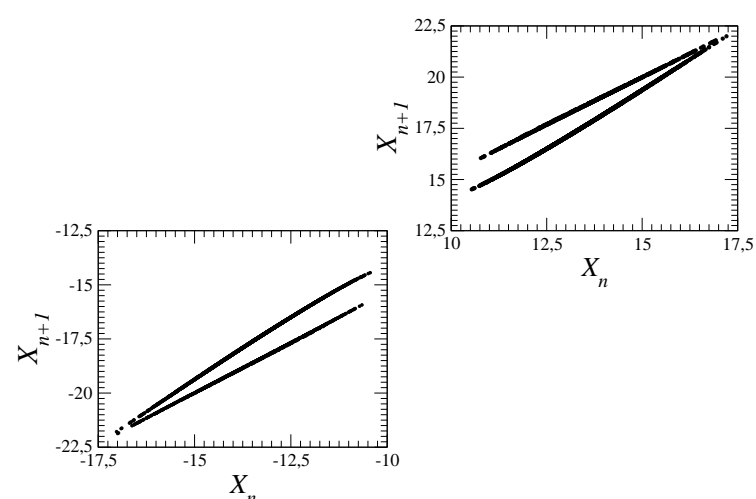

(a) Poincaré section

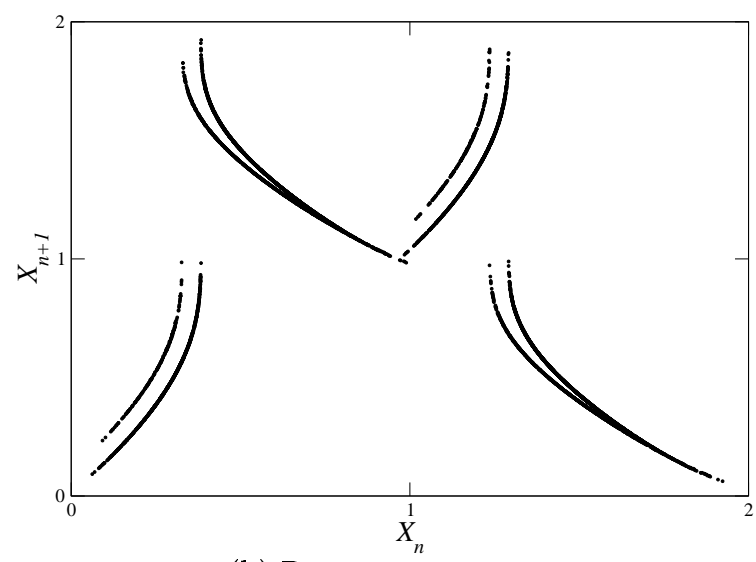

(b) Return map on $u_{n}$

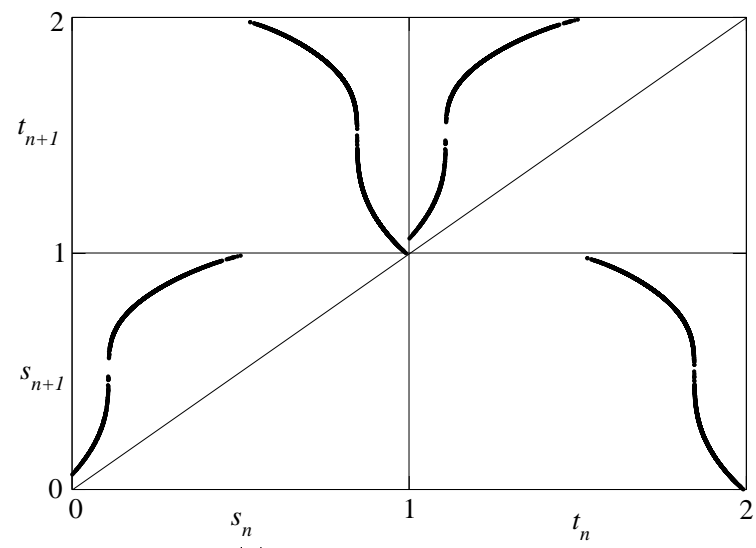

(c) Return map on $s_{n}$

FIG. 11: (a) Intersections of the chaotic attractor with the two components of the global Poincaré surface of section $X=Y= \pm 9.5, \beta=3.8$. (b) First return plot for the intersections of the attractor with the global Poincaré surface of section. (c) Return map based on parameterization of the intersection shown in (b), with $s$ parameterizing the left-hand intersection and $t$ parameterizing the right-hand intersection. Parameter values: $(\sigma, \nu, R, \Lambda)=(10,8 / 3,74.667,3.2)$. 
original covering attractors, was close to $\ln 2$.

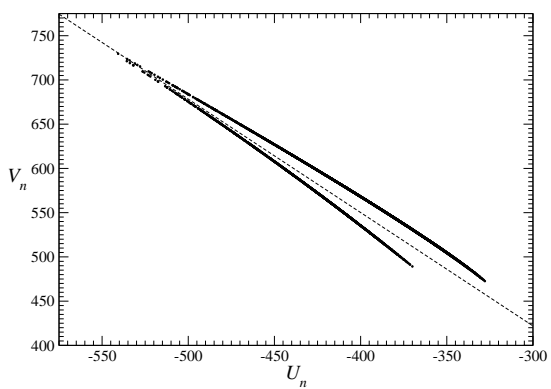

(a) Poincaré section

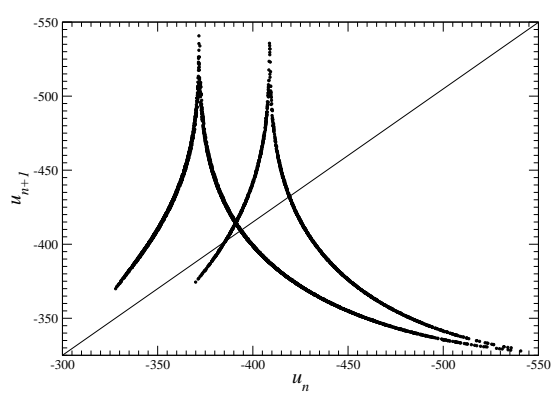

(b) Return map on $u_{n}$

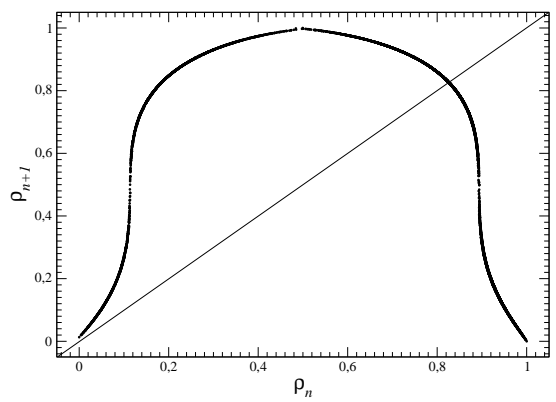

(c) Return map on $s_{n}$

FIG. 12: For the image attractor shown in Fig. 8 with $\beta=7.9$ : (a) Intersection with a Poincaré section; (b) Return map based on coordinate $u_{n}$. The separation between the two cusps increases with $\beta$; (c) Return map based on parameterization of the intersection shown in (a). Parameter values: $(\sigma, \nu, R, \Lambda)=$ $(10,8 / 3,74.667,3.2)$.

\section{SUMMARY AND CONCLUSIONS}

We have studied a four-dimensional dynamical system that is designed to model the behavior of a self-exciting homopolar Faraday disk dynamo. This system is essentially the Lorenz system with feedback. The coupling is implemented by a fourth variable, $U$, which is driven by the $X$ variable of the Lorenz system, and feeds back into the dynamics by driving $X$ through a coupling parameter of strength $\beta$. We studied the dynamics generated in the range $0 \leq \beta \leq 7.9$ for fixed control parameter values of the Lorenz system $(\sigma, \nu, R)=(10,8 / 3,74.667)$. The variable $U$ is damped with $\Lambda=3.2$. The one-parameter $(\beta)$ family of chaotic attractors that is generated is threedimensional.

In order to carry out topological analyses, we have attempted to find three-dimensional embeddings of the attractor. Of the two obvious embeddings constructed by reducing the dimension through reduction of the number of variables, one mapping $(X, Y, Z, U) \rightarrow(X, Y, Z)$ fails to provide an embedding for intermediate values of $\beta$. Furthermore, the branched manifolds describing the projected attractors in the small- $\beta$ and large- $\beta$ limits are mirror images of each other: they are both of Lorenz type with rotation symmetry. The other projection, $(X, Y, Z, U) \rightarrow(X, Y, U)$, does provide an embedding for all values of $\beta$ studied. The branched manifold describing all attractors in this projection is a Lorenz branched manifold with inversion symmetry. The branched manifolds obtained in these two projections are related by "group continuation" [10]. They describe the same stretching and tearing mechanism.

Similar tests for embeddings and topological analyses were carried out in several other faithful $(1 \leftrightarrow 1)$ and $2 \rightarrow 1$ image representations of the dynamics. In general, carrying out the analysis in the image is simpler than in the cover since the global Poincaré surface of section has only one connected component. We found generally that an almost complete dynamics on two symbols was present for all values of $\beta$. For fixed $\beta$ all embeddings (even mappings that were not embeddings) exhibited an identical spectrum of orbits (or image orbits). When an embedding existed the table of linking numbers obtained from these orbits was compatible with a branched manifold. When an embedding did not exist, this compatibility did not exist. In such cases, orbits whose linking numbers were incorrect were those that visited regions of the mapped chaotic attractor undergoing self-intersections.

\section{ACKNOWLEDGMENTS}

R. G. thanks CNRS for the invited position at CORIA for 2006-2007.

\section{REFERENCES}

[1] R. Hide, A. C. Skeldon \& D. J. Acheson, Proc. R. Soc. Lond. A, 452, 1369-1395, 1996.

[2] R. Hide \& I. M. Moroz, Physica D, 134, 287-301, 1999.

[3] I. M. Moroz, Int. J. Bif. \& Chaos, 13, 147-161, 2003.

[4] I. M. Moroz, Int. J. Bif. \& Chaos, 14, 2885-2892, 2004.

[5] I. M. Moroz, Nonlinear Dynamics, 41, 191-210, 2005.

[6] I. M. Moroz, Proc. R. Soc. Lond. A, 463, 113-130, 2007. 
[7] K.A. Robbins, Math. Proc. Cambridge Philos. Soc., 82, 309-325, 1977.

[8] R. Gilmore, Revs. Mod. Phys. 70, 1455 (1998).

[9] R. Gilmore and M. Lefranc, The Topology of Chaos, NY: Wiley, 2002.

[10] R. Gilmore \& C. Letellier, The Symmetry of Chaos, NY: Oxford, 2006.

[11] C. Letellier \& R. Gilmore. Phys. Rev. E, 63, 16206, 2001.

[12] R. Gilmore and C. Letellier, Phys. Rev. E67, 134104 (2003).
[13] C. Letellier \& L. A. Aguirre, Chaos, 12, 549-558, 2002.

[14] C. Letellier \& L. A. Aguirre, Phys. Rev. E, 72, $056202,2005$.

[15] G. Byrne, R. Gilmore \& C. Letellier, Phys. Rev. E, 70, 056214, 2004.

[16] C. Letellier, T. D. Tsankov, G. Byrne, and R. Gilmore, Phys. Rev. E72, 026212 (2005).

[17] N. Romanazzi, M. Lefranc, and R. Gilmore (unpublished). 